\title{
TSFD: Two Stage Frame Dropping for Scalable Video Transmission over Data Networks *
}

\author{
Bing Zheng \\ Dept. of Electrical and Computer Eng. \\ University of Dayton \\ Dayton, OH 45469 \\ zhengbin@flyernet.udayton.edu
}

\author{
Mohammed Atiquzzaman \\ School of Computer Science \\ University of Oklahoma \\ Norman, OK 73019 \\ atiq@ieee.org
}

\begin{abstract}
Scalable video transmission is used to adjust the rate of video depending on the level of network congestion. Previous studies on scalable video transmission of MPEG over ATM ABR service required major changes in the network protocols, and did not provide methods to determine the ABR connection parameters. In this paper, we propose a new scalable video transmission scheme which does not require major changes in network protocols. In our proposed scheme, frames are dynamically dropped either by the source or the network depending on the level of network congestion.
\end{abstract}

\section{Introduction}

Video transmission requires a large bandwidth from the network. During periods of network congestion, the bit rate of video needs to be scaled dynamically depending on the level of network congestion $[1,2]$. This paper deals with a new video scaling scheme for interactive video on demand systems.

The authors in [2] investigated layer based scalable MPEG video transmission scheme over ATM networks. However, the schemes have to be implemented at the video source coder. The authors in $[1,3]$ studied static layer based and slice based scalable MPEG transmission over the ATM VBR/ABR hybrid service. Unfortunately, their schemes need modification of the the standard AAL5 protocol, and hence is not suitable for practical deployment. Moreover, they did not provide any algorithm to choose an optimal value of Minimum Cell Rate (MCR) to ensure QoS. The objective of this paper is to develop a scalable scheme for transmitting stored MPEG video over a bandwidth limited channel without requiring major changes of the standard network protocols.

We propose a novel Two Stage Frame Dropping (TSFD) Scheme for scalable MPEG transmission over an ATM ABR service. In addition to frames being dropped by the server in the case of network congestion, the server also marks low priority frames to be dropped by the network in the case of severe congestion. An important contribution of this paper is that the value of MCR is chosen by a combination of the

\footnotetext{
*This work was supported by a fellowship from DAGSI
}

bit rate and burstiness of video. Our proposed TFSD scheme is based on encapsulating video frames with priority information which is used to drop frames by the network during congestion. The scheme requires no major change of network protocols.

The effect of MPEG video GoP on the client buffer size has been also analyzed. A general framework has have been developed to determine the client buffer size for no overflow at the client.

The rest of this paper is organized as follows. The principle of TSFD is presented in Section 2, followed by Section 3 where we develop a framework to determine the optimal buffer size. Numerical results are presented in Section 4, followed by conclusions in Section 5 .

\section{Two Stage Frame Dropping (TSFD)}

The Two Stage Frame Dropping (TSFD) scheme proposed in this paper consists of two parts. The first is the dynamic priority encapsulation and frame discarding procedure under different network congestion. The second is the adaptive choice of MCR by taking into consideration the burstiness and bit rate of video. Notations used throughout the paper are given below.

$f$ : MPEG video frame rate in frames/second;

$n$ : Distance between I frames for $\mathrm{MmNn}$ GoP;

$m$ : Distance between $\mathrm{P}$ frames for $\mathrm{MmNn}$ GoP;

$X_{I}, X_{P}, X_{B}$ : Average size of I, $\mathrm{P}$ and $\mathrm{B}$ frames in bits;

$x_{I}$ : I frame size in bits;

$\beta_{I}$ : Average bit rate of I frames defined as $X_{I} f$;

$\beta_{P}$ : Average bit rate of $\mathrm{P}$ frames defined as $X_{P} f$;

$\beta_{B}$ : Average bit rate of $\mathrm{B}$ frames defined as $X_{B} f$;

$E_{0}[\beta]$ : Average bit rate of video stream with I, P and B frames;

$E_{1}[\beta]$ : Average bit rate for video stream with $\mathrm{I}$ and $\mathrm{P}$ frames;

$E_{2}[\beta]$ : Average bit rate for video stream with only I frames; 


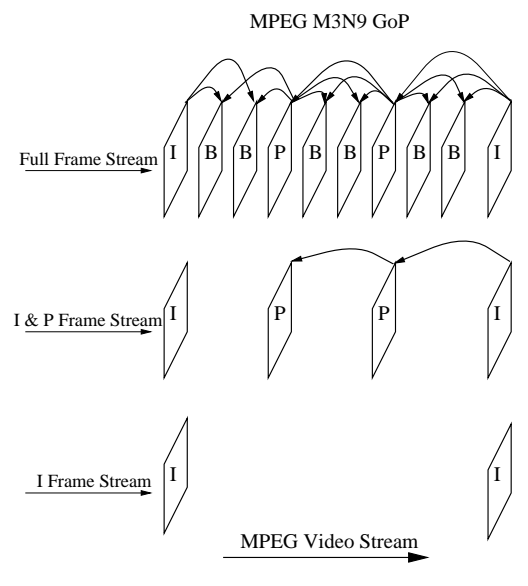

Figure 1: Three video streams having different combinations of frame types.

$k$ : Speed factor for fastforward / fastbackward (FFW/FBW) operation in a video on demand system;

$A C R, M C R$ : The available and minimum cell rates respectively during playback;

$C_{c}$ : Critical value of client buffer size;

$\rho$ : The ratio of MCR to $E_{2}[\beta]$;

$T_{d}$ : Fixed Round Trip Time (FRTT) from server to client;

$\tau_{f}$ : Duration of FFW/FBW operation;

$\tau_{c}$ : Duration of network congestion;

$b=\frac{\beta_{I}}{\beta_{B}}:$ Burst coefficient for MPEG;

$n_{1}, n_{2}$ : The expected number of requests before the server gets the FFW/FBW and playback bandwidth respectively.

\subsection{Principle of TSFD}

Loss of I, P, or B frames have different effects on video quality. I frame is the reference frame which is most important, $\mathrm{P}$ and $\mathrm{B}$ frames are less important because they contribute mainly to the improvement of space and time resolution. Therefore, in case of network congestion, $\mathrm{B}$ and $\mathrm{P}$ frames can be discarded to reduce the bandwidth requirement of video. As shown in Figure 1, different combination of frame types require different amounts of bandwidth. Our proposed TSFD scheme can be described as follows:

- During normal playback, frames are dropped either by the server and/or by the network depending on the level of congestion.

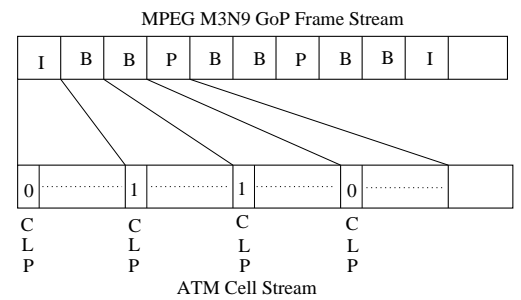

Figure 2: Priority encapsulation in the TSFD scheme.

- The assignment of the Cell Loss Priority (CLP) bit (in the ATM cell header) by the server is done as follows. If the bandwidth offered by the network meets the requirements for transporting full frame video stream, the server does not drop frames, and sets $\mathrm{CLP}=1$ for $\mathrm{B}$ frames (Figure 2). However, if the bandwidth offered by the network meets the requirements for transporting only I and $\mathrm{P}$ frames, the server discards the $\mathrm{B}$ frames, and assigns $\mathrm{CLP}=1$ to $\mathrm{P}$ frames. If the bandwidth offered by the network just meets the requirements for transporting I frames, the server discards B and P frames; it randomly sets some of the I frames with CLP $=1$ allowing the network to drop those I frames in the case of congestion.

- In the FFW or FBW mode, the server discards all $\mathrm{B}$ and $\mathrm{P}$ frames, and sends only I frames to reduce the bandwidth.

- During FFW/FBW operation, the server accepts whatever rate is available from the network, but keeps sending in rate RM cells to request a rate which is no less than the required rate for FFW/FBW operation.

\subsection{Adaptive Choice of MCR}

Once an ABR connection is set up, any bandwidth request which is higher than the MCR is approved by the network with some probability. We describe below an algorithm to adaptively choose a value of MCR. From Figure 1, the average bit rate corresponding to the three frame streams can be expressed as:

$$
\begin{aligned}
E_{0}[\beta] & =\frac{\beta_{I}+\beta_{P}(n / m-1)+\beta_{B} n / m(m-1)}{n}(1) \\
E_{1}[\beta] & =\frac{\beta_{I}+\beta_{P}(n / m-1)}{n} \\
E_{2}[\beta] & =\frac{\beta_{I}}{n}
\end{aligned}
$$

To ensure adequate quality of video at the client, the value of MCR should be between $E_{2}[\beta]$ and $E_{0}[\beta]$, and is given by.

$$
M C R=\rho E_{2}[\beta]
$$

where $\rho$ has three different values corresponding to the three different streams.

$$
\rho_{\text {low }}=1
$$




BEGIN Connection setup
Set $M C R=E_{0}[\beta] / \rho_{\text {low }}$
Send ABR Connection Setup Signal
If Returned $M C R<M C R$, then Set $M C R=$
$E_{0}[\beta] / \rho_{\text {middle }}$
Send ABR Connection Setup Signal
If Returned $M C R<M C R$, then Set $M C R=$
$E_{0}[\beta] / \rho_{\text {high }}=$
Send ABR Connection Setup Signal
If Returned $M C R<M C R$, then Wait a random
time period and try again
END

Figure 3: The algorithm for choosing MCR.

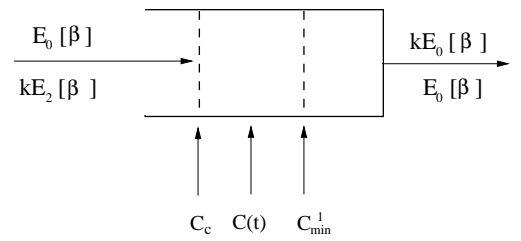

Figure 4: Illustration of $C_{\text {min }}^{1}$ and $C_{c}$ at client buffer.

$$
\begin{aligned}
\rho_{\text {middle }} & =1+\frac{1}{b} \frac{\beta_{P}}{\beta_{B}}\left(\frac{n}{m}-1\right) \\
\rho_{\text {high }} & =1+\frac{1}{b} \frac{\beta_{P}}{\beta_{B}}\left(\frac{n}{m}-1\right)+\frac{1}{b} \frac{n}{m}(m-1)
\end{aligned}
$$

$\rho$ reflects the contributions from the $\mathrm{B}$ and $\mathrm{P}$ frames, and hence is a measure of the burstiness of video. The algorithm to choose MCR is shown in Figure 3.

\section{Client Buffer Size}

We define the critical client buffer size $C_{c}$ as the size below which the client buffer overflows with a high probability. To determine $C_{c}$, we first need to determine the minimum client buffer fill level which is defined as the buffer level at which there is no starvation at the client.

\subsection{Minimum Client Buffer Fill Level}

The value of the $M C R$ that is accepted by the network may be lower than the average rate of the video which gives rise to the following two cases.

- Case 1: In FFW/FBW operation, there should be no starvation at the client buffer;

- Case 2: In normal playback, there should be no starvation at the client buffer during network congestion.

\subsubsection{Case 1: FFW/FBW Operation}

As shown in Figure 4, we assume that at time $t$, the client sends a FFW/FBW request to the video server. The client starts its FFW/FBW operation, consuming video data at a rate $k E_{0}[\beta]$. We also assume that FFW/FBW lasts for a time duration $\tau_{f}$. During this period, the client will consume $Q_{\text {out }}^{1}=\int_{t}^{t+\tau_{f}} k E_{0}[\beta] d t$ amount of data. On other hand, because of network congestion and propagation delay, the client will not immediately receive data at the $\mathrm{FFW} / \mathrm{FBW}$ rate after sending the FFW/FBW request. The delay consists of three parts: $T_{d} / 2$ for the $\mathrm{FFW} / \mathrm{FBW}$ request to arrive at the server, $n_{1} T_{d}$ to obtain the requested bandwidth of $k E_{2}[\beta]$, and $T_{d} / 2$ for the data to arrive at the client. During this time, the data input rate to client buffer is still at the rate of $E_{0}[\beta]$. The input data is denoted by $Q_{i n 1}^{1}=\int_{t}^{t+\left(n_{1}+1\right) T_{d}} E_{0}[\beta] d t$. $Q_{i n 2}^{1}=\int_{t+\left(n_{1}+1\right) T_{d}}^{t+\tau_{f}} k E_{2}[\beta] d t$ is the amount of input data at FFW/FBW speed. Therefore, for no starvation at the client buffer, the amount of data consumed by the client must be equal to or less than the sum of the arriving data and the previously stored data in the buffer:

$$
C(t)+Q_{i n 1}^{1}+Q_{i n 2}^{1}-Q_{o u t}^{1} \geq 0
$$

where $C(t)$ is client buffer fill level at time $t$. By writing Equation (8) in average value form:

$$
\begin{array}{r}
C(t)+\left(n_{1}+1\right) T_{d} E_{0}[\beta]+\left(\tau_{f}-\left(n_{1}+1\right) T_{d}\right) k E_{2}[\beta]- \\
\left(n_{1}+1\right) T_{d} k E_{0}[\beta]-\left(\tau_{f}-\left(n_{1}+1\right) T_{d}\right) k E_{0}[\beta] \geq 0
\end{array}
$$

Therefore, to prevent the client buffer from starvation during the FFW/FBW operation, the client buffer must have a minimum fill level of $C_{m i n}^{1}$. Note that $C_{\text {min }}^{1}$ is the value of $C(t)$ for the minimum case in Equation (9).

$$
\begin{gathered}
C_{\min }^{1} \geq(k-1)\left(n_{1}+1\right) T_{d} E_{0}[\beta]+ \\
k\left(\tau_{f}-\left(n_{1}+1\right) T_{d}\right)\left(1-\frac{1}{\rho_{h i g h}}\right) E_{0}[\beta]
\end{gathered}
$$

\subsubsection{Case 2: Heavy Network Congestion}

In case of heavy network congestion, $A C R=M C R$. Assume that the congestion duration lasts for a period of $\tau_{c}$. To prevent the client buffer from underflow, a minimum fill level of $C_{\min }^{2}$ is required to compensate the difference between the low video data input rate and high data consumption rate from the client buffer as shown in Figure 5.

In the worst case, only after the end of heavy congestion, can the server obtain the required normal playback rate after an average of $n_{2}$ requests. During the time period $\tau_{c}+\left(n_{2}+0.5\right) T_{d}$, the amount of input data to the client buffer is $Q_{i n}^{2}=$ $\int_{t}^{t+\tau_{c}+\left(n_{2}+0.5\right) T_{d}} E_{2}[\beta] d t$, and the amount of data consumed by the client from the buffer is $Q_{\text {out }}^{2}=$ 


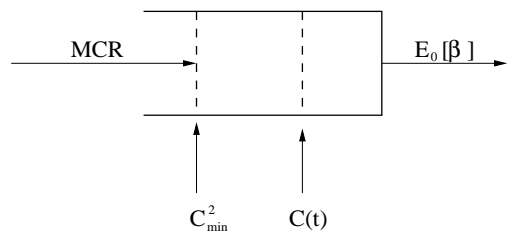

Figure 5: Illustration of $C_{\min }^{2}$ at the client buffer

$$
\begin{array}{r}
\int_{t}^{t+\tau_{c}+\left(n_{2}+0.5\right) T_{d}} E_{0}[\beta] d t . \text { Therefore: } \\
C(t)+Q_{\text {in }}^{2}-Q_{\text {out }}^{2} \geq 0
\end{array}
$$

$C_{\min }^{2}$ and $C_{\min }$ are obtained from the following conditions:

$$
\begin{gathered}
C_{\text {min }}^{2} \geq\left(1-\frac{1}{\rho_{\text {high }}}\right)\left(\tau_{c}+\left(n_{2}+1\right) T_{d}\right) E_{0}[\beta] \\
C_{\text {min }}=\max \left(C_{\text {min }}^{1}, C_{\text {min }}^{2}\right)
\end{gathered}
$$

It can be seen that the minimum client buffer fill is directly related to the network congestion, video rate, and the FRTT of the channel.

\subsection{Critical Client Buffer Size}

As illustrated in Figure 4, after the FFW/FBW operation is finished, the client will send a signal to the server to restore its outgoing data to normal playback rate. It takes $T_{d} / 2$ for the server to receive the signal and another $T_{d} / 2$ for the client to receive the data sent from the server at the normal playback rate. So, $C_{f}$ is buffer space required by the client to accumulate this fluctuation.

$$
C_{f}=T_{d}\left(\frac{k}{\rho_{\text {high }}}-1\right) E_{0}[\beta]
$$

From Equations (10), (12), (13) and (14), the critical client buffer size $C_{c}$ at the client is:

$$
C_{c}=C_{f}+C_{\text {min }}
$$

The client buffer overflow probability can be estimated from the critical client buffer size requirement.

\section{Results and Discussion}

An MPEG video stream with typical values of $X_{I}=400 \mathrm{kbits}, X_{P}=200 \mathrm{kbits}$ and $X_{B}=80 \mathrm{kbits}$ has been used to get the critical client buffer size $C_{c}$ for different MPEG GoPs. dino [4], an MPEG sequence with following parameters has been used in this study. GoP Pattern: M3N12 IBBPBBPBBPBB; Frame Rate: 24 frames/second; Quantizer Scale: 10 for I frame, 14 for P frame and 18 for B frame; Resolution: $384 * 288$ pels, 12 bit color information; Mean Frame Size: 13078 bits; Burst Coefficient b: $>$ 9.1; Peak Bit Rate: 1.01 Mbps; Mean Bit Rate: 0.33 Mbps.

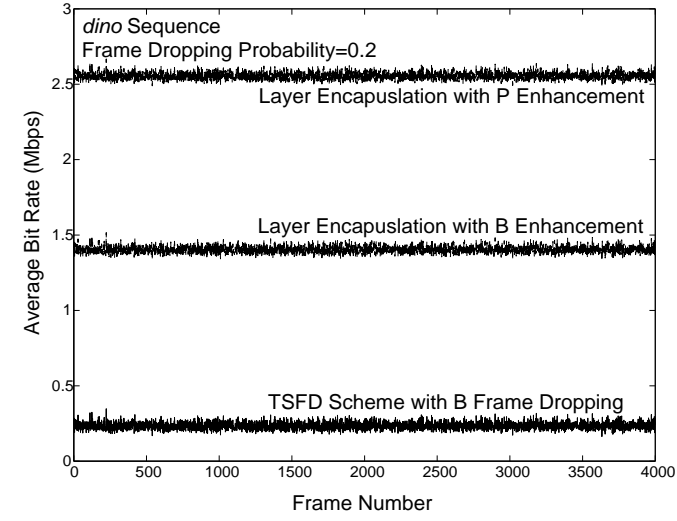

Figure 6: Average bit rate corresponding to three encapsulating scheme for sequence dino.

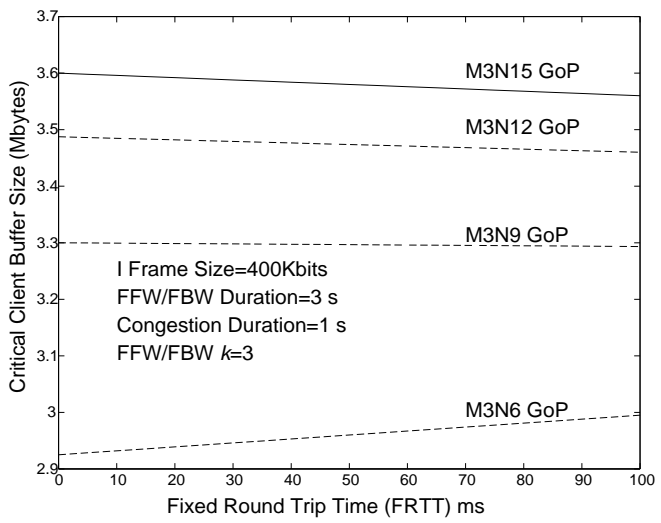

Figure 7: Critical Client buffer size $C_{c}$ versus fixed round trip time (FRTT).

Figure 6 shows the average bit rate for sequence dino corresponding to TSFD scheme with $\mathrm{B}$ frame dropping, layer based priority encapsulation with $\mathrm{P}$ enhancement, and layer based priority encapsulation with B enhancement. TSFD scheme requires the lowest bandwidth.

The critical client buffer size $C_{C}$ versus FRTT is shown in Figure 7 for different GoP. As expressed in Equations (10), (12), (13), (14) and (15), the client buffer size depends on FFW/FBW time $\tau_{f}$, video parameter $\rho_{\text {high }}$ and $E_{0}[\beta]$, and FFW/FBW speed factor $k$. So, the $T_{d}$ has different effect on the critical client buffer size for different GoP. High GoP pattern needs a larger buffer size than low GoP pattern since it gets more contribution from $\mathrm{B}$ and $\mathrm{P}$ frames.

The critical client buffer size $C_{c}$ versus the FFW/FBW duration with a constant FRTT is shown in Figure 8. As the FFW/FBW time increases, the buffer size increases linearly for all MPEG GoP structures. This is because the longer the FFW/FBW operation, the higher the data consumed by the client. 


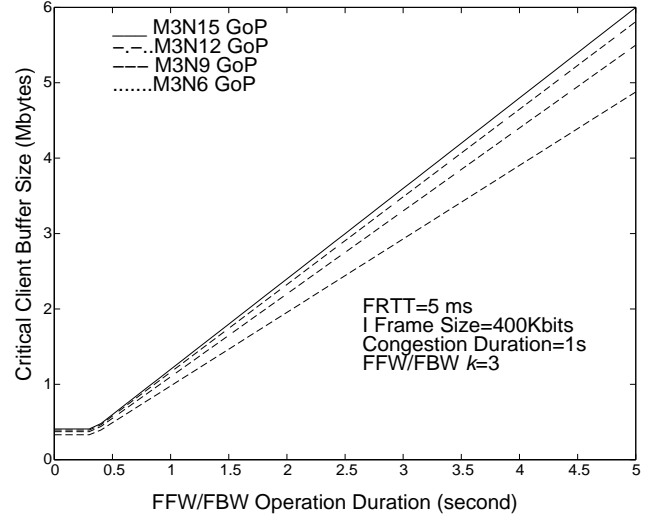

Figure 8: Critical Client buffer size $C_{c}$ versus FFW/FBW time.

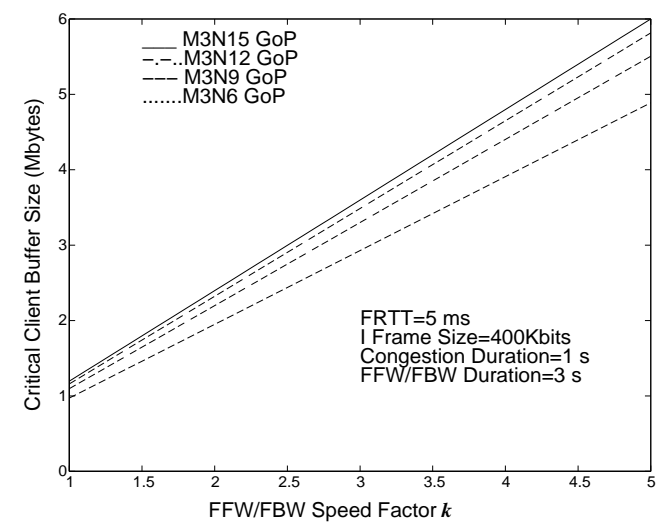

Figure 9: Critical Client buffer size $C_{c}$ versus FFW/FBW speed factor $k$.

Higher GoP pattern has a larger $\rho_{\text {high }}$ which implies a higher data rate. So, the critical client buffer size for a higher GoP pattern has a larger increasing slope. Note that the fixed part at the beginning is coming from the effect of Equation (14).

Figure 9 shows the critical client buffer size as a function of FFW/FBW speed factor $k$. As expected from Equation (15), the critical client buffer size increases linearly with the FFW/FBW speed factor. Similarly, because a higher GoP pattern has a larger $\rho_{\text {high }}$ and data rate, it also has a larger slope.

Figure 10 shows the critical client buffer size as a function of the network congestion duration. When network congestion lasts for a period of time shorter than nine seconds, the critical client buffer size remains constant. Only when the congestion time become longer than nine seconds, the critical client buffer size increases linearly with the congestion time.

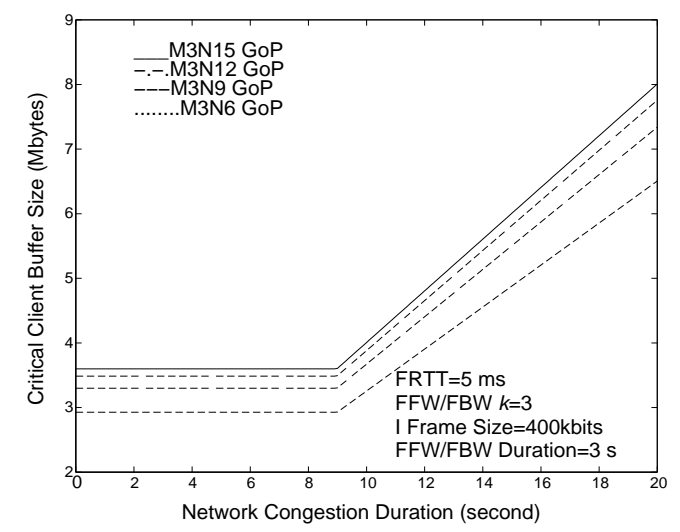

Figure 10: Critical Client buffer size $C_{c}$ versus network congestion duration.

\section{Conclusion}

In this paper, we have proposed a Two Stage Frame Dropping (TSFD) for scalable MPEG transmission over an ATM ABR service. Our scheme adaptively sets the value of MCR, and also adjusts the video rate dynamically according to the network congestion. We have also developed a statistical model to determine the client buffer size. We conclude that the client buffer size has a linear relationship with the FFW/FBW operation time and the FFW/FBW speed factor. The client buffer size increases linearly with long term network congestion, and is insensitive to network size, burstiness of the video, and short term network congestion. We have shown that the client buffer requirement depends on the MPEG GoP structure; usually, a large GoP pattern requires a large client buffer. The results in this paper can be used by system and network designers to determine the optimal buffer size and fine tune the network parameters to allow video on demand systems over the ATM ABR service.

\section{References}

[1] Ahmed Mehaoua and Raouf Boutaba, "Performance analysis of cell discarding techniques for best effort video communications over ATM networks," Computer Networks and ISDN Systems, vol. 29, no. 17-18, pp. 1999-2019, February 1998.

[2] Wenjun Luo and Magda El Zarki, "Quality control for VBR video over ATM networks," IEEE Journal on Selected areas in Communications, vol. 15, no. 6, pp. 1029-1039, August 1997.

[3] A. Mehaoua, R. Boutaba, and G. Pujolle, "An extended priority data partition scheme for MPEG video connection over ATM," IEEE Symposium on Computers and Communication'98, Alexandria, Egypt, pp. 6267, July 1998.

[4] O. Rose, "Statistical properties of MPEG video traffic and their impact on traffic modeling in ATM system," research report, Institute of Computer Science, University of Wurzburg, Feb 1995. 